\title{
Accepting Zero in the Ultimatum Game Does Not Reflect Selfish Preferences
}

\author{
Gianandrea Staffiero $^{\text {a }}$, Filippos Exadaktylos ${ }^{\text {b* }} \&$ Antonio M. Espín ${ }^{c}$
}

a. Universitat Pompeu Fabra, Spain. Department of Economics and Business

b. BELIS, Murat Sertel Center for Advanced Economic Studies, Istanbul Bilgi

University, Santral Campus 34060 Istanbul, Turkey

c. Universidad de Granada, Departamento de Teoría e Historia Económica, Spain

* Corresponding author. Email: ph.exadaktylos@gmail.com.

First Version (“Accepting Zero in the Ultimatum Game: Selfish Nash Response?”):

January 2013.

This Version: June 2013

\begin{abstract}
The study shows that subjects who set their minimum acceptable offer equal to zero in an ultimatum game (UG) are the most generous dictators in a dictator game. The finding implies that interpreting indiscriminately the acceptance of low UG offers as payoff maximization can be misleading and suggests that altruism and/or the desire to maximize welfare are to a large extent behind.
\end{abstract}

JEL Classifications: C93, C91, D03, C70

Keywords: ultimatum game, dictator game, altruism, social welfare, selfishness, otherregarding preferences 


\section{Introduction}

In the ultimatum game (UG) (Güth et al. 1982), a proposer issues an offer on how to divide a sum of money to a responder who may either accept, and thus implement the proposer's split, or reject at the cost of destroying the whole endowment. Based on the canonical assumption of selfish rationality, a responder should accept any positive amount since a rejection would leave her with no earnings at all. In case the proposal leaves her with exactly a zero-payoff, a selfish responder should be indifferent between accepting and rejecting it. Technically speaking, her weakly dominant strategies consist in setting her minimum acceptable offer (MAO) either to zero or to the smallest positive amount ( $\varepsilon$ ). If both options are available, the selfish responder's choice should result from randomization between the two.

Subjects' behavior in UG experiments, however, does not comply with these theoretical predictions since unequal offers are very often rejected by responders, even in one-shot games (see Camerer 2003). Many economics models have been proposed in order to incorporate the possibility that some agents are not purely selfish but exhibit "otherregarding preferences" (see the review of Fehr and Schmidt 2006). Along these lines, the same behavioral pattern is often compatible with more than one set of assumptions. UG rejections for example have been rationalized on the basis of responders' inequity aversion (Fehr and Schmidt 1999, Bolton and Ockenfels 2000), spiteful preferences (Kirchsteiger 1994), negative reciprocity (Rabin 1993) or norm-compliance (López-Pérez 2008).

Relatively less attention has been devoted to the motives behind those strategies that do follow standard predictions since they were automatically considered to be confirming the theory and its main assumptions. ${ }^{1}$ In particular, in this paper we focus on responders who accept any proposal in an ultimatum game, setting their minimum acceptable offer (MAO) at zero. While this behavior could result from the randomization between the two payoff-maximizing strategies (i.e. setting MAO at zero or at the smallest positive amount,

\footnotetext{
${ }^{1}$ Indeed a non-negligible portion of the subjects in experiments (between $20 \%$ and $50 \%$ depending on the study) behave according to the predictions of a fully-rational, fully-selfish agent (see e.g. Fehr and Schmidt 2006).
} 
$\varepsilon)$, it also implies that the opponent's payoff is maximized and the "pie" remains intact and can thus be compatible with either altruism (Becker 1974) or social welfare maximization (Charness and Rabin 2002). An agent driven by such motives will strictly prefer MAO=0 to the rest of possible MAOs, including $\mathrm{MAO}=\varepsilon$.

We combine MAO responses and offers in a dictator game (DG) (Forsythe et al. 1994). In the DG, the possibility of rejection is removed, so that the amount first movers (“dictators") pass to second movers denotes pro-social behavior, free of any strategic concerns (which are present in UG offers and thus make them difficult to interpret). If "MAO-zero players" (those accepting any offer, including zero) make lower offers as dictators than the rest of responders, then the unwillingness to reject low offers will likely be stemming from selfish motivations. Moreover, if this is the case, MAO-zero and "MAO$\varepsilon$ players" (those accepting anything but zero) should behave similarly in the DG.

\section{Experimental procedures}

We analyze data from two similar experiments. The city experiment took place between November and December 2010 in the city of Granada, Spain, with a representative sample of 835 citizens between 16 and 91 years old who were interviewed in their own apartments by university students. The lab experiment was conducted in the Granada Lab of Experimental Economics EGEO at the University of Granada during October 2011. During 27 sessions data were collected from a sample of 659 university students.

In both cases, subjects completed the exact same survey-experiment comprised of a dictator and an ultimatum game. ${ }^{2}$ All subjects (i) decided how to split the pie in the DG, (ii) made an offer on how to split the pie in the UG (in both cases multiples of 2 , between 0 and 20 euros, were used) and, (iii) employing the strategy method, decided whether to accept or reject any possible UG proposal (limited to offers between 0 and 10). The benefits of the strategy method, introduced in the UG by Mitzkewitz and Nagel (1993), are obvious in

\footnotetext{
${ }^{2}$ They also played a trust game. The exact design of the survey-experiment has been previously reported and described in detail in Exadaktylos et al. (2013).
} 
terms of data collection, especially given that low offers - necessary for our analysis - tend to be rare. ${ }^{3}$ The order in which decisions were presented was randomized across participants. Double-blinded procedures were used in both experiments. Subjects were also informed that after the experiment a random selection would determine who among them (one out of ten subjects) would receive a payment and which game would determine the amount.

\section{Results}

$8.37 \%$ (54 out of 645 ) of the subjects in the lab and $12.81 \%$ (102 out of 796 ) in the city chose $\mathrm{MAO}=0$ as UG responders. ${ }^{4}$ In panel a (b) of Figure 1 we show the mean DG giving in the lab (city) according to the MAO chosen as responder in the UG.

In the lab, MAO-zero players gave on average 9.70 out of their endowment of 20 in the DG. In the city, MAO-zero players gave 8.82 on average in the DG. While students in the lab were on average more generous than normally observed, ${ }^{5}$ in both samples MAOzero players gave significantly more than the rest of responders: In the lab, the mean ( \pm s.e.m.) DG giving among subjects with a positive MAO (2 or more) decreases to $8.09 \pm 0.14$ ( $P=0.0005$; two-tailed Mann-Whitney test), while in the city it decreases to $7.72 \pm 0.16(P=0.0161)$. Interestingly, MAO- $\varepsilon$ players (i.e. those who set $\mathrm{MAO}=2)$ were the greediest dictators in both samples (7.41 in the lab and 7.02 in the city) giving significantly less than MAO-zero players (lab: $P=0.0003$, city: $P=0.0171$ ).

\footnotetext{
${ }^{3}$ A recent review by Brandts and Charness (2011) shows that results with direct responses are similar to the ones observed with the use of the strategy method.

${ }^{4}$ Data from 14 subjects in the lab and from 39 subjects in the city were removed due to inconsistency problems (e.g. accepting a UG proposal and rejecting a higher one) or to missing values in any of the decisions.

5 Students in our sample were "pseudo-volunteers" in the sense that experiments were conducted during a class. Pseudo-volunteer students have been found to be more generous than the students who volunteer in experiments (the standard subject pool) (Eckel and Grossman 2000). Thus, our result might have been affected by this particular recruitment system. Nevertheless, we do not see any reason to think that this can influence the relationship between the behavior in ultimatum and dictator games.
} 

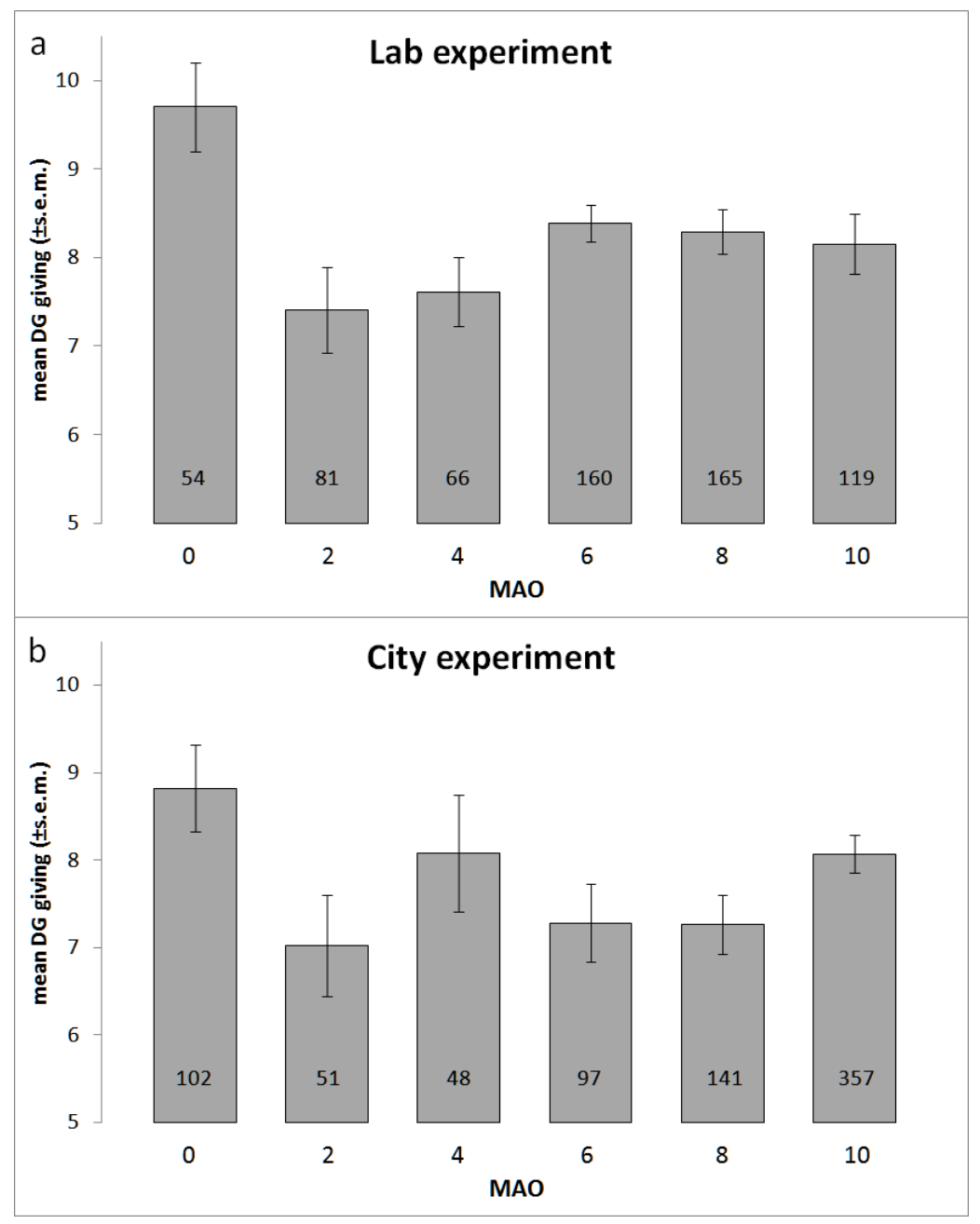

Figure 1. Mean DG giving ( \pm s.e.m.) by MAO groups (numbers inside the bars indicate $n$ ).

\section{Discussion}

These results indicate that players who accept any offer in the UG, including the one that leaves them with zero payoff, are far from selfish. In fact generous dictators' giving in combination with not punishing in the UG can be explained in terms of altruism, whereby the opponent's payoff enters positively in the utility function. The same also follows if a player is concerned with social welfare, that immediately implies setting $\mathrm{MAO}=0$ (destroying the pie obviously reduces welfare) and, probably, making generous dictator offers as well (if the marginal increase in welfare decreases with wealth, a fifty-fifty 
distribution is socially optimal). ${ }^{6}$ This is in line with the observation of de Oliveira and Eckel (2012) who, in light of recent empirical evidence (Brañas-Garza et al. 2006, Chen and Tang 2009), suggest that the acceptance of low offers could be importantly motivated by generosity, rather than self-interest.

Our findings therefore contradict the idea that the responders whose behavior does not deviate from standard Nash predictions are payoff-maximizing individuals. An immediate corollary is that those who do deviate, sacrificing their payoffs in order to punish, are not necessarily "less selfish". This implies that interpreting responders' decisions in terms of a conflict between payoff maximization and other-regarding preferences, as usually proposed, could be misleading. For instance, in an influential paper, Knoch et al. (2006) suggest that rejections require that responders "resist the economic temptation to accept these [unfair] offers" (pg. 829), given that disrupting subjects' selfcontrol with brain stimulation techniques was found to increase the likelihood of acceptance. Note however, that an offer of, say 4 euros $(20 \%$ of the pie, as used by Knoch et al. 2006), would have been accepted by all subjects with $M A O \leq 4$ in our setting, i.e. by a combination of extremely different types of players, including both the most and the least generous individuals (MAO-zero and MAO- $\varepsilon$ players, respectively). Indeed, in the lab (city), 27\% (51\%) of all individuals who accept 4 euros are MAO-zero players. So, depending on the distribution of types in their sample, the acceptance of a low offer could respond more to a "pro-social temptation" than to an "economic temptation". Thus, our results indicate that not only the rejection but also the acceptance of low offers in the UG can reflect other-regarding preferences.

\footnotetext{
${ }^{6}$ Note that the Fehr and Schmidt's (1999) model of inequity aversion cannot account for our findings since a restriction is imposed so that one cannot be more averse to advantageous than to disadvantageous inequality $(\alpha \geq \beta)$. That means that someone who is generous in the DG should also reject low UG offers.
} 


\section{Acknowledgments}

The authors are thankful to Pablo Brañas-Garza for useful comments. The usual disclaimer applies. Financial support from the Spanish Ministry of Science and Innovation (SEJ201017049/ECON) and the Regional Government of Andalusia (PO7-SEJ-02547) are also gratefully acknowledged. FE acknowledges the post-doctorate fellowship granted by the Scientific and Technological Research Council of Turkey (TUBITAK).

\section{References}

Becker, G.S. 1974. A theory of social interactions. Journal of Political Economy 82, 10631093.

Bolton, G.E., Ockenfels, A. 2000. ERC: a theory of equity, reciprocity, and competition. American Economic Review 90, 166-193.

Brandts, J., Charness, G. 2011. The strategy versus the direct-response method: a first survey of experimental comparisons. Experimental Economics 14, 375-398.

Brañas-Garza, P., Cobo-Reyes, R., Domínguez, A. 2006. Si él lo necesita: Gypsy fairness in Vallecas. Experimental Economics 9, 253-264.

Camerer, C.F. 2003. Behavioral game theory: experiments in strategic interaction. Princeton: Princeton University Press.

Charness, G., Rabin, M. 2002. Understanding social preferences with simple tests. Quarterly Journal of Economics 117, 817-869.

Chen, K., Tang, F.-F. 2009. Cultural differences between Tibetans and ethnic Han Chinese in ultimatum bargaining experiments. European Journal of Political Economy 25, 78-84.

de Oliveira, A., Eckel, C.C. 2012. Heterogeneity in ultimatum bargaining: the role of information, individual characteristics, and emotions. In G.E. Bolton, R.T.A. Croson (eds.) 
The Oxford handbook of economic conflict resolution. New York: Oxford University Press, pp. 295-312.

Eckel, C.C., Grossman, P.J. 2000. Volunteers and pseudo-volunteers: the effect of recruitment method in dictator experiments. Experimental Economics 3, 107-120.

Exadaktylos, F., Espín, A.M., Brañas-Garza, P. 2013. Experimental subjects are not different. Scientific Reports 3, 1213.

Fehr, E., Schmidt, K.M. 1999. A theory of fairness, competition and cooperation. Quarterly Journal of Economics 114, 817-868.

Fehr, E., Schmidt, K.M. 2006. The economics of fairness, reciprocity and altruism experimental evidence and new theories. In S.-C. Kolm, J.M. Ythier (eds.) Handbook of the economics of giving, altruism and reciprocity, vol. 1. Amsterdam: Elsevier, pp. 615-691.

Forsythe, R., Horowitz, J.L., Savin, N.E., Sefton, M. 1994. Fairness in simple bargaining experiments. Games and Economic Behavior 6, 347-369.

Güth, W., Schmittberger, R., Schwarze, B. 1982. An experimental analysis of ultimatum bargaining. Journal of Economic Behavior \& Organization 3, 367-388.

Kirchsteiger, G. 1994. The role of envy in ultimatum games. Journal of Economic Behavior \& Organization 25, 373-389.

Knoch, D., Pascual-Leone, A., Meyer, K., Treyer, V., Fehr, E. 2006. Diminishing reciprocal fairness by disrupting the right prefrontal cortex. Science 314, 829-832.

López-Pérez, R. 2008. Aversion to norm-breaking: a model. Games and Economic Behavior 64, 237-267.

Mitzkewitz, M., Nagel, R. 1993. Experimental results on ultimatum games with incomplete information. International Journal of Game Theory 22, 171-198.

Rabin, M. 1993. Incorporating fairness into economics and game theory. American Economic Review 83, 1281-1302. 